\title{
Joint secrecy over the $K$-Transmitter Multiple Access Channel
}

\author{
Yanling Chen*, O. Ozan Koyluoglu ${ }^{\dagger}$ and A. J. Han Vinck* \\ * Institute of Digital Signal Processing, University of Duisburg-Essen, Germany. \\ $\dagger$ Department of Electrical and Computer Engineering, The University of Arizona. \\ Email: \{yanling.chen, han.vinck\}@uni-due.de, ozan@email.arizona.edu.
}

\begin{abstract}
This paper studies the problem of secure communication over a $K$-transmitter multiple access channel in the presence of an external eavesdropper, subject to a joint secrecy constraint (i.e., information leakage rate from the collection of $K$ messages to an eavesdropper is made vanishing). As a result, we establish the joint secrecy achievable rate region. To this end, our results build upon two techniques in addition to the standard information-theoretic methods. The first is a generalization of Chia-El Gamal's lemma on entropy bound for a set of codewords given partial information. The second is to utilize a compact representation of a list of sets that, together with properties of mutual information, leads to an efficient FourierMotzkin elimination. These two approaches could also be of independent interests in other contexts.
\end{abstract}

\section{INTRODUCTION}

For the problem of reliably communicating independent messages over a multiple access channel (MAC), Ahlswede [1] first studied the 2-transmitter and 3-transmitter cases and determined the respective capacity regions; whilst Liao 22 considered the general $K$-transmitter MAC and fully characterized its capacity region.

Inspired by the pioneering works of Wyner [3] and Csiszár and Körner 4 that studied the information theoretic secrecy of a point-to-point communication in the presence of an external eavesdropper, a MAC with an external eavesdropper was first introduced in [5]. In particular, 5 focused on a degraded Gaussian MAC with $K$-transmitters and established achievable rate regions subject to a pre-specified secrecy measure; while a discrete memoryless 2-transmitter MAC with an external eavesdropper was considered in 6 . Further works on MAC with an external eavesdropper include but not limited to 7 [10]. However, its secrecy capacity region, even for the 2transmitter case, still remains an open problem.

In this paper, we consider the secure communication over a $K$-transmitter MAC subject to the joint secrecy constraint (i.e., information leakage rate from the collection of $K$ messages to an eavesdropper is made vanishing), the channel model of which is shown in Fig. 1] As a general result, we establish a joint secrecy achievable rate region.

This work is supported in part by DFG Grant CH 601/2-1 and NSF award CNS-1617335.

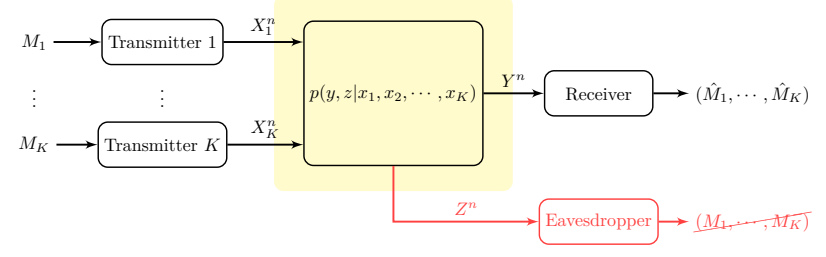

Fig. 1: $K$-transmitter DM-MAC with an eavesdropper.

The rest of the paper is organized as follows. Section II introduces the system model and presents necessary definitions; Section III gives the general conditional entropy bound. The bound plays an important role in establishing the joint secrecy rate region that is given in Section III To enhance the flow of the paper, some details for the Fourier-Motzkin elimination are relegated to Appendix A

\section{Preliminaries}

Consider a discrete memoryless MAC (DM-MAC) with $K$ transmitters, one legitimate receiver, and one passive eavesdropper, which is defined by $p\left(y, z \mid x_{1}, x_{2}, \cdots, x_{K}\right)$. The transmitter $i$, aims to send message $m_{i}$, to the legitimate receiver, where $i \in \mathcal{K}=\{1,2, \cdots, K\}$. Suppose that $x_{i}^{n}$ is the channel input at transmitter $i$, and the channel outputs at the legitimate receiver and eavesdropper are $y^{n}$ and $z^{n}$, respectively. By the discrete memoryless nature of the channel (without any feedback), we have

$$
p\left(y^{n}, z^{n} \mid x_{1}^{n}, \cdots, x_{K}^{n}\right)=\prod_{i=1}^{n} p\left(y_{i}, z_{i} \mid x_{1, i}, \cdots, x_{K, i}\right) .
$$

A $\left(2^{n R_{1}}, 2^{n R_{2}}, \cdots, 2^{n R_{K}}, n\right)$ secrecy code $\mathcal{C}_{n}$ for the DMMAC consists of

- $K$ message sets $\mathcal{M}_{1}, \mathcal{M}_{2}, \cdots, \mathcal{M}_{K}$, where $m_{i} \in \mathcal{M}_{i}=$ $\left[1: 2^{n R_{i}}\right]$ for $i \in \mathcal{K}$;

- $K$ encoders each assigning a codeword $x_{i}^{n}$ to message $m_{i}$ for $i \in \mathcal{K}$; and

- One decoder at the legitimate receiver that declares an estimate of $\left(m_{1}, m_{2}, \cdots, m_{K}\right)$ say $\left(\hat{m}_{1}, \hat{m}_{2}, \cdots, \hat{m}_{K}\right)$ or an error to the received sequence $y^{n}$.

In this paper, for a list of random variables $W_{j}$ for $j \in \mathcal{K}$, and a fixed $\mathcal{J} \subseteq \mathcal{K}$, we denote $W_{\mathcal{J}}=\left\{W_{i} \mid i \in \mathcal{J}\right\}$. Assume that the messages $M_{\mathcal{K}}$ are uniformly distributed 
over their corresponding message sets. Therefore, we have $R_{i}=\frac{1}{n} H\left(M_{i}\right)$, for $i \in \mathcal{K}$. Denote the average probability of decoding error at the legitimate receiver as $P_{e}^{n}\left(\mathcal{C}_{n}\right)=\operatorname{Pr}\left\{\bigcup_{i \in \mathcal{K}}\left\{M_{i} \neq \hat{M}_{i}\right\} \mid \mathcal{C}_{n}\right\}$. Define the joint information leakage rate to the eavesdropper by $R_{L, \mathcal{K}}\left(\mathcal{C}_{n}\right)=$ $\frac{1}{n} I\left(M_{\mathcal{K}} ; Z^{n} \mid \mathcal{C}_{n}\right)$. The rate pair $\left(R_{1}, R_{2}, \cdots, R_{K}\right)$ is said to be achievable under the joint secrecy constraint, if there exists a sequence of $\left(2^{n R_{1}}, 2^{n R_{2}}, \cdots, 2^{n R_{K}}, n\right)$ codes $\left\{\mathcal{C}_{n}\right\}$ such that

$$
\begin{aligned}
P_{e}^{n}\left(\mathcal{C}_{n}\right) & \leq \epsilon_{n}, \\
R_{L, \mathcal{K}}\left(\mathcal{C}_{n}\right) & \leq \tau_{n}, \\
\lim _{n \rightarrow \infty} \epsilon_{n} & =0 \text { and } \quad \lim _{n \rightarrow \infty} \tau_{n}=0 .
\end{aligned}
$$

Recall that $\mathcal{K}=\{1,2, \cdots, K\}$. We have the following definition and lemmas.

Definition 1. The indicator vector of a subset $\mathcal{T}$ of set $\mathcal{K}$, denoted by $1_{\mathcal{T}}$, is a $1 \times K$ vector, with its $i$-th element equal to 1 if $i \in \mathcal{T}$ and 0 otherwise, for $1 \leq i \leq K$.

For instance, for $K=5, \mathcal{K}=\{1,2,3,4,5\}$ and $\mathcal{T}=$ $\{1,3,5\}$, we have $1_{\mathcal{T}}=\left[\begin{array}{lllll}1 & 0 & 1 & 0 & 1\end{array}\right]$, and $1_{\emptyset}=\left[\begin{array}{lllll}0 & 0 & 0 & 0 & 0\end{array}\right]$.

Let $\left\{\mathcal{T}_{i} \mid 1 \leq i \leq t\right\}$ be a list of $t$ subsets of $\mathcal{K}$.

Definition 2. The presence vector of $\left\{\mathcal{T}_{i} \mid 1 \leq i \leq t\right\}$ is defined to be $\mathrm{t}_{\#}=\sum_{i=1}^{t} 1_{\mathcal{T}_{i}}$, which counts the number of presences of each element of $\mathcal{K}$ over $\left\{\mathcal{T}_{i} \mid 1 \leq i \leq t\right\}$.

Definition 3. A compact form of the element rearrangement for $\left\{\mathcal{T}_{i} \mid 1 \leq i \leq t\right\}$ is defined to be $\left\{\mathcal{T}_{t, i}^{*} \mid 1 \leq i \leq t\right\}$, where $\mathcal{T}_{t, i}^{*}$ contains the elements that present at least $i$ times from all these $t$ subsets, i.e.,

$$
\mathcal{T}_{t, i}^{*}=\bigcup_{\left\{j_{1}, \cdots, j_{i}\right\} \subseteq[1: t]}\left(\bigcap_{k=1}^{i} \mathcal{T}_{j_{k}}\right) .
$$

Clearly, $\mathcal{T}_{t, t}^{*} \subseteq \mathcal{T}_{t, t-1}^{*} \subseteq \cdots \subseteq \mathcal{T}_{t, 1}^{*}$. And, $\mathcal{T}_{t, i}^{*}=\emptyset$ for $i>$ $t_{\max }$, where $t_{\max }$ is the largest element of $\mathrm{t}_{\#} \cdot$ So $\left\{\mathcal{T}_{t, i}^{*} \mid 1 \leq\right.$ $\left.i \leq t_{\max }\right\}$ is the compact form without the empty sets.

Lemma 4. $\mathrm{t}_{\#}=\sum_{i=1}^{t} 1_{\mathcal{T}_{i}}=\sum_{i=1}^{t} 1_{\mathcal{T}_{t, i}^{*}}=\sum_{i=1}^{t_{\max }} 1_{\mathcal{T}_{t, i}^{*}}$.

For instance, for $K=3, \mathcal{K}=\{1,2,3\}$ and $\left\{\mathcal{T}_{i} \mid 1 \leq i \leq 3\right\}$ with $\mathcal{T}_{1}=\{1\}, \mathcal{T}_{2}=\{1,2\}, \mathcal{T}_{3}=\{2,3\}$. We have $\mathrm{t}_{\#}=$ $(2,2,1), t_{\max }=2, \mathcal{T}_{3,1}^{*}=\{1,2,3\}, \mathcal{T}_{3,2}^{*}=\{1,2\}, \mathcal{T}_{3,3}^{*}=\emptyset$.

Lemma 5. Given two lists of sets $\left\{\mathcal{T}_{1 i} \mid 1 \leq i \leq t_{1}\right\}$ and $\left\{\mathcal{T}_{2 i} \mid 1 \leq i \leq t_{2}\right\}$, elements of which are $t_{1}$ and $t_{2}$ subsets of set $\mathcal{K}$, respectively, if they share the same presence sequence $\mathrm{t}_{\#}$, then they also share the same compact form of the element rearrangement without empty sets.

\section{CONDitional ENTROPy BOUnd}

In this section, we give the conditional entropy bound, which is a generalization of Chia-El Gamal's lemma 11, Lemma 1] on entropy bound for a set of conditionally independent codewords given partial information.
Lemma 6. Let $R_{v, i} \geq 0$ for $i \in \mathcal{K}, \epsilon>0$, and $\left(Q, V_{1}, \cdots, V_{K}, Z\right) \sim p(q) \cdot \prod_{i \in \mathcal{K}} p\left(v_{i} \mid q\right) \cdot p\left(z \mid v_{1}, \cdots, v_{K}\right)$. Let $Q^{n}$ be a random sequence and each $q^{n}=(q(1), \cdots, q(n))$ distributed according to $\prod_{t=1}^{n} p(q(t))$. For $i \in \mathcal{K}$, let $V_{i}^{n}\left(l_{i}\right), l_{i} \in\left[1: 2^{n R_{v, i}^{t=1}}\right]$, be a set of random sequences that are conditionally independent given $Q^{n}$ and each $v_{i}^{n}=\left(v_{i}(1), \cdots, v_{i}(n)\right)$ distributed according to $\prod_{t=1}^{n} p\left(v_{i}(t) \mid q(t)\right)$, and let $\mathcal{C}$ be the codebook of $\left(Q^{n}, V_{1}^{n}(1), \cdots, V_{K}^{n}\left(2^{n R_{v, K}}\right)\right)$. Let $L_{i}$ be the random index of $V_{i}^{n}$, for $i \in \mathcal{K}$, with an arbitrary probability mass function. Then, if $\operatorname{Pr}\left\{\left(Q^{n}, V_{1}^{n}\left(L_{1}\right), \cdots, V_{K}^{n}\left(L_{K}\right), Z^{n}\right) \in\right.$ $\left.\mathcal{T}_{\epsilon}^{n}\left(Q, V_{1}, \cdots, V_{K}, Z\right)\right\} \rightarrow 1$ as $n \rightarrow \infty$ and

$$
\sum_{j \in \mathcal{J}} R_{v, j} \geq I\left(V_{\mathcal{J}} ; Z \mid Q\right), \quad \forall \mathcal{J} \subseteq \mathcal{K}
$$

there exists a $\delta_{n}(\epsilon) \rightarrow 0$ as $\epsilon \rightarrow 0$ and $n \rightarrow \infty$, such that for $n$ sufficiently large, $H\left(L_{1}, \cdots, L_{K} \mid Z^{n}, Q^{n}, \mathcal{C}\right) \leq$ $n\left[\sum_{j \in \mathcal{K}} R_{v, j}-I\left(V_{\mathcal{K}} ; Z \mid Q\right)\right]+n \delta_{n}(\epsilon)$.

Proof: Given $z^{n}$, let us define $\mathcal{L}$ as the set of indices $\left(l_{1}, l_{2}, \cdots, l_{K}\right)$ such that

$$
\left(q^{n}, v_{1}^{n}\left(l_{1}\right), \cdots, v_{K}^{n}\left(l_{K}\right), z^{n}\right) \in \mathcal{T}_{\epsilon}^{n}\left(Q, V_{1}, \cdots, V_{K}, Z\right) .
$$

First we show that the expected size of this list, over all randomly generated codebooks, is upper bounded by

$$
\mathbb{E}(|\mathcal{L}|) \leq 1+\sum_{i=1}^{2^{K}-1} 2^{n\left[\mathrm{I}_{i}+\delta(\epsilon)\right]}
$$

where $\mathrm{I}_{i}=\sum_{j \in \mathcal{J}_{i}} R_{v, j}-I\left(V_{\mathcal{J}_{i}} ; Z \mid V_{\mathcal{J}_{i}^{c}}, Q\right)$. Here, $\left\{\mathcal{J}_{i} \mid i \in[1:\right.$ $\left.\left.2^{K}-1\right]\right\}$ are the $2^{K}-1$ non-empty subsets of $\mathcal{K}$, and $\mathcal{J}_{i}^{c}=\mathcal{K} \backslash \mathcal{J}_{i}$ for $i \in\left[1: 2^{K}-1\right]$. Note that

$$
\begin{aligned}
\mathbb{E}(|\mathcal{L}|)= & \operatorname{Pr}\left\{\left(L_{1}, \cdots, L_{K}\right) \in \mathcal{L}\right\} \\
& +\sum_{\left(l_{1}, \cdots, l_{K}\right) \neq\left(L_{1}, \cdots, L_{K}\right)} \operatorname{Pr}\left\{\left(l_{1}, \cdots, l_{K}\right) \in \mathcal{L}\right\},
\end{aligned}
$$

where $\left(L_{1}, \cdots, L_{K}\right)$ are the true indices chosen by the sources. Since $\operatorname{Pr}\left\{\left(q^{n}, v_{1}^{n}\left(L_{1}\right), \cdots, v_{K}^{n}\left(L_{K}\right), z^{n}\right) \in\right.$ $\left.\mathcal{T}_{\epsilon}^{n}\left(Q, V_{1}, \cdots, V_{K}, Z\right)\right\} \rightarrow 1$ as $n \rightarrow \infty$, the 1 st term tends to 1 as $n \rightarrow \infty$. As for the 2 nd term, we can distinguish $\left(2^{K}-1\right)$ cases according to the values of $\left(l_{1}, l_{2}, \cdots, l_{K}\right)$. More specifically, for each $\mathcal{J}_{i}, i \in\left[1: 2^{K}-1\right]$, we consider the following case:

- $l_{j} \neq L_{j}$ for $j \in \mathcal{J}_{i}$, and $l_{j}=L_{j}$ for $j \in \mathcal{J}_{i}^{c}$.

In this case, in total there are at most $2^{n \sum_{j \in \mathcal{J}_{i}}}$ possible $\left(l_{1}, \cdots, l_{K}\right)$. By the joint typicality lemma, we can upper bound $\operatorname{Pr}\left\{\left(l_{1}, \cdots, l_{K}\right) \in \mathcal{L}\right\}$ by

$$
2^{-n I\left(V_{\mathcal{J}_{i}} ; V_{\mathcal{J}_{i}^{c}}, Z \mid Q\right)+n \delta(\epsilon)} \stackrel{(a)}{=} 2^{-n I\left(V_{\mathcal{J}_{i}} ; Z \mid V_{\mathcal{J}_{i}}{ }^{c}, Q\right)+n \delta(\epsilon)},
$$

where $(a)$ is due to the fact that $V_{\mathcal{J}_{i}}$ and $V_{\mathcal{J}_{i}^{c}}$ are conditionally independent given $Q$. 
Therefore, in this case, there are at most $2^{n\left[\mathrm{I}_{i}+\delta(\epsilon)\right]}$ number of $\left(l_{1}, l_{2}, \cdots, l_{K}\right)$ falling in the list $\mathcal{L}$.

Summing up all the numbers of $\left(l_{1}, l_{2}, \cdots, l_{K}\right)$ falling in the list $\mathcal{L}$ over all these $\left(2^{K}-1\right)$ cases, we prove 6 .

Furthermore, define the indicator variable $E=1$ if $\left(L_{1}, \cdots, L_{K}\right) \in \mathcal{L}$, and $E=0$ otherwise. We have

$$
\begin{aligned}
& H\left(L_{1}, \cdots, L_{K} \mid Z^{n}, Q^{n}, \mathcal{C}\right) \leq H\left(L_{1}, \cdots, L_{K}, E \mid Z^{n}, Q^{n}, \mathcal{C}\right) \\
& \quad \leq H(E)+H\left(L_{1}, \cdots, L_{K} \mid Z^{n}, Q^{n}, E, \mathcal{C}\right) \\
& \quad \stackrel{(b)}{\leq} 1+H\left(L_{1}, \cdots, L_{K} \mid Z^{n}, Q^{n}, E=1, \mathcal{C}\right) \\
& \quad+\operatorname{Pr}\{E=0\} H\left(L_{1}, \cdots, L_{K} \mid \mathcal{C}\right),
\end{aligned}
$$

where $(b)$ follows from the fact that $H(E) \leq 1$ since $E$ is a binary random variable; $\operatorname{Pr}\{E=1\} \leq 1$ and conditioning does not increase the entropy.

Since $\operatorname{Pr}\left\{\left(q^{n}, v_{1}^{n}\left(L_{1}\right), \cdots, v_{K}^{n}\left(L_{K}\right), z^{n}\right) \in \mathcal{T}_{\epsilon}^{n}\left(Q, V_{1}, \cdots\right.\right.$, $\left.\left.V_{K}, Z\right)\right\} \rightarrow 1$ as $n \rightarrow \infty$, then $\operatorname{Pr}\{E=0\}=$ $\operatorname{Pr}\left\{\left(L_{1}, L_{2}, \cdots, L_{K}\right) \notin \mathcal{L}\right\}$ can be made arbitrarily small as $n \rightarrow \infty$. Next,

$$
\begin{aligned}
& H\left(L_{1}, \cdots, L_{K} \mid Z^{n}, Q^{n}, E=1, \mathcal{C}\right) \\
& \stackrel{(c)}{=} H\left(L_{1}, \cdots, L_{K}\left|Z^{n}, Q^{n}, E=1, \mathcal{C}, \mathcal{L},\right| \mathcal{L} \mid\right) \\
& \leq H\left(L_{1}, \cdots, L_{K}|E=1, \mathcal{L},| \mathcal{L} \mid\right) \\
& =\sum_{l \in \operatorname{supp}(|\mathcal{L}|)} \operatorname{Pr}\{|\mathcal{L}|=l\} H\left(L_{1}, \cdots, L_{K}|E=1, \mathcal{L},| \mathcal{L} \mid=l\right) \\
& \stackrel{(d)}{\leq} \sum_{l \in \operatorname{supp}(|\mathcal{L}|)} \operatorname{Pr}\{|\mathcal{L}|=l\} \log _{2}(l) \\
& =\mathbb{E}\left(\log _{2}(|\mathcal{L}|)\right) \stackrel{(e)}{\leq} \log _{2}(\mathbb{E}(|\mathcal{L}|)) \\
& \stackrel{(f)}{\leq} n \max _{\max }\left\{0, \max _{i \in\left[1: 2^{K}-1\right]} \mathrm{I}_{i}\right\}+K+n \delta(\epsilon) \\
& \stackrel{(g)}{\leq} n\left[\sum_{j \in \mathcal{K}} R_{v, j}-I\left(V_{\mathcal{K}} ; Z \mid Q\right)\right]+K+n \delta(\epsilon),
\end{aligned}
$$

where $(c)$ follows from the fact that $\mathcal{L}$ and $|\mathcal{L}|$ are functions of the output $Z^{n}$, given $\mathcal{C}$ and $Q^{n} ;(d)$ is due to the fact that, knowing $E=1$, the sent indices $\left(L_{1}, L_{2}, \cdots, L_{K}\right)$ belong to the list $\mathcal{L}$ and the uncertainty is upper bounded by the $\log$ cardinality of the list; $(e)$ is by Jensen's inequality; $(f)$ is by (6) along with an application of the logsum-exp inequality: $\log _{2}\left(\sum_{x \in \mathcal{X}} 2^{x}\right) \leq \max _{x \in \mathcal{X}} x+\log _{2}(|\mathcal{X}|)$; and $(g)$ follows if the rates satisfies (5), i.e.: $\sum_{j \in \mathcal{J}} R_{v, j} \geq$ $I\left(V_{\mathcal{J}} ; Z\right), \forall \mathcal{J} \subseteq \mathcal{K}$. This, along with previous remarks yields the desired inequality (by defining $\delta_{n}(\epsilon)$ to be the arbitrary small term $\mathcal{O}(\epsilon)+(K+1) / n)$.

\section{Achievable Rate Region}

In this section, we give an achievable joint secrecy rate region of the $K$-transmitter DM-MAC with an external eavesdropper. This result recovers the joint secrecy result for $K=2$ in [12, Theorem 2], which improves [6. (8)] with channel prefixing as demonstrated in 12 .
Theorem 7. An achievable joint secrecy rate region of the $K$-transmitter DM-MAC with an external eavesdropper is given by the union of non-negative rate pairs $\left(R_{1}, R_{2}, \cdots, R_{K}\right)$ that are defined by the followings:

$$
\sum_{j \in \mathcal{J}} R_{j} \leq I\left(V_{\mathcal{J}} ; Y \mid V_{\mathcal{J}^{c}}, Q\right)-I\left(V_{\mathcal{J}} ; Z \mid Q\right), \quad \forall \mathcal{J} \subseteq \mathcal{K}
$$

where the union is over input probability distributions that factor as $p(q) \prod_{i \in \mathcal{K}} p\left(v_{i} \mid q\right) p\left(x_{i} \mid v_{i}\right)$.

Proof: Fix $p(q)$ and $p\left(v_{i} \mid q\right), p\left(x_{i} \mid v_{i}\right)$ for $i \in \mathcal{K}$. Generate a random sequence $q^{n}$, where $p\left(q^{n}\right)=\prod_{t=1}^{n} p(q(t))$ with each entry chosen as i.i.d. $p(q)$. The sequence $q^{n}$ is given to every node in the system.

Codebook generation: For $i \in \mathcal{K}$, to construct codebook $\mathcal{C}_{i}$, randomly generate $2^{n\left[R_{i}+R_{i, r}\right]}$ i.i.d. sequences $v_{i}^{n}\left(m_{i}, m_{i, r}\right)$, with $\left(m_{i}, m_{i, r}\right) \in\left[1: 2^{n R_{i}}\right] \times\left[1: 2^{n R_{i, r}}\right]$, each with probability $p\left(v_{i}^{n} \mid q^{n}\right)=\prod_{t=1}^{n} p\left(v_{i}(t) \mid q(t)\right)$. Every node in the network knows these codebooks. Denote the overall codebook as $\mathcal{C}$.

Encoding: For $i \in \mathcal{K}$, to send message $m_{i}$, transmitter $i$ randomly and uniformly chooses $m_{i, r} \in\left[1: 2^{n R_{i, r}}\right]$ and finds $v_{i}^{n}\left(m_{i}, m_{i, r}\right)$. Then, given the codeword $v_{i}^{n}\left(m_{i}, m_{i, r}\right)$, it generates $x_{i}^{n}$ according to $\sum_{t=1}^{n} p\left(x_{i}(t) \mid v_{i}(t)\right)$ and transmits this sequence to the channel.

Decoding: The legitimate receiver, upon receiving $y^{n}$, finds $v_{1}^{n}\left(\hat{m}_{1}, \hat{m}_{1, r}\right), v_{2}^{n}\left(\hat{m}_{2}, \hat{m}_{2, r}\right), \ldots, v_{K}^{n}\left(\hat{m}_{K}, \hat{m}_{K, r}\right)$ such that $\left(v_{1}^{n}\left(\hat{m}_{1}, \hat{m}_{1, r}\right), v_{2}^{n}\left(\hat{m}_{2}, \hat{m}_{2, r}\right), \cdots, v_{K}^{n}\left(\hat{m}_{K}, \hat{m}_{K, r}\right), y^{n}\right)$ is jointly typical.

Analysis of the error probability of decoding: Consider the expected value of the error probability of decoding over the ensemble of random codes $\mathcal{C}$, i.e., $P_{e}=\mathbb{E}\left[P_{e}(\mathcal{C})\right]$. Note that here $\mathcal{C}$ denotes the random variable that represents the randomly generated codebook that adhere to the above scheme. From the decoding analysis for the multiple access channel, see, e.g., [13], $P_{e}$ can be made approximately zero as $n \rightarrow \infty$ if

$$
\sum_{j \in \mathcal{J}}\left[R_{j}+R_{j, r}\right] \leq I\left(V_{\mathcal{J}} ; Y \mid V_{\mathcal{J}^{c}}, Q\right), \quad \forall \mathcal{J} \subseteq \mathcal{K} .
$$

Analysis of joint secrecy: For the joint secrecy as defined in (3), we show in the following that $\mathbb{E}\left[R_{L, \mathcal{K}}(\mathcal{C})\right] \leq \tau_{n}$. To this end, we show that $H\left(M_{\mathcal{K}} \mid Z^{n}, Q^{n}, \mathcal{C}\right) \geq n \sum_{i \in \mathcal{K}} R_{i}-n \tau_{n}$, as this implies $I\left(M_{\mathcal{K}} ; Z^{n} \mid \mathcal{C}\right) \leq I\left(M_{\mathcal{K}} ; Z^{n}, Q^{n} \mid \mathcal{C}\right) \leq n \tau_{n}$.

$$
\begin{aligned}
& H\left(M_{\mathcal{K}} \mid Z^{n}, Q^{n}, \mathcal{C}\right)=H\left(M_{\mathcal{K}}, Z^{n} \mid Q^{n}, \mathcal{C}\right)-H\left(Z^{n} \mid Q^{n}, \mathcal{C}\right) \\
& =H\left(M_{\mathcal{K}}, M_{\mathcal{K}, r} \mid Q^{n}, \mathcal{C}\right)+H\left(Z^{n} \mid M_{\mathcal{K}}, M_{\mathcal{K}, r}, Q^{n}, \mathcal{C}\right) \\
& \quad-H\left(Z^{n} \mid Q^{n}, \mathcal{C}\right)-H\left(M_{\mathcal{K}, r} \mid M_{\mathcal{K}}, Z^{n}, Q^{n}, \mathcal{C}\right) \\
& \stackrel{(a)}{=} H\left(M_{\mathcal{K}}, M_{\mathcal{K}, r} \mid Q^{n}, \mathcal{C}\right)+H\left(Z^{n} \mid V_{1}^{n}, \cdots, V_{K}^{n}, M_{\mathcal{K}}, M_{\mathcal{K}, r}, Q^{n}, \mathcal{C}\right) \\
& \quad-H\left(Z^{n} \mid Q^{n}, \mathcal{C}\right)-H\left(M_{\mathcal{K}, r} \mid M_{\mathcal{K}}, Z^{n}, Q^{n}, \mathcal{C}\right) \\
& \stackrel{(b)}{=} n \sum_{i \in \mathcal{K}}\left[R_{i}+R_{i, r}\right]+H\left(Z^{n} \mid V_{1}^{n}, V_{2}^{n}, \cdots, V_{K}^{n}, Q^{n}, \mathcal{C}\right)
\end{aligned}
$$




$$
\begin{aligned}
& \quad-H\left(Z^{n} \mid Q^{n}, \mathcal{C}\right)-H\left(M_{\mathcal{K}, r} \mid M_{\mathcal{K}}, Z^{n}, Q^{n}, \mathcal{C}\right) \\
& \stackrel{(c)}{\geq} \sum_{i \in \mathcal{K}}\left[R_{i}+R_{i, r}\right]-I\left(V_{1}^{n}, \cdots, V_{K}^{n} ; Z^{n} \mid Q^{n}, \mathcal{C}\right) \\
& \quad-n\left[\sum_{i \in \mathcal{K}} R_{i, r}-I\left(V_{\mathcal{K}} ; Z \mid Q\right)+\varepsilon_{n}\right] \stackrel{(d)}{\geq} n \sum_{i \in \mathcal{K}} R_{i}-n \tau_{n},
\end{aligned}
$$

where $(a)$ follows from the fact that $V_{1}^{n}, \cdots, V_{K}^{n}$ are functions of $\left(M_{1}, M_{1, r}\right), \cdots,\left(M_{K}, M_{K, r}\right)$, respectively, given $Q^{n}$ and $\mathcal{C}$; $(b)$ follows from the fact that $H\left(M_{\mathcal{K}}, M_{\mathcal{K}, r} \mid Q^{n}, \mathcal{C}\right)=n \sum_{i \in \mathcal{K}}\left[R_{i}+R_{i, r}\right]$, and given $Q^{n}$ and $\mathcal{C},\left(M_{\mathcal{K}}, M_{\mathcal{K}, r}\right) \rightarrow\left(V_{1}^{n}, V_{2}^{n}, \cdots, V_{K}^{n}\right) \rightarrow Z^{n}$ forms a Markov chain; (c) follows from Lemma 6 (with $R_{v, i}=R_{i, r}$ for $i \in \mathcal{K})$ by requiring (5):

$$
\sum_{j \in \mathcal{J}} R_{j, r} \geq I\left(V_{\mathcal{J}} ; Z \mid Q\right), \quad \forall \mathcal{J} \subseteq \mathcal{K} .
$$

$(d)$ is due to the fact that $I\left(V_{1}^{n}, \cdots, V_{K}^{n} ; Z^{n} \mid Q^{n}, \mathcal{C}\right) \leq$ $n\left[I\left(V_{1}, \cdots, V_{K} ; Z \mid Q\right)+\varepsilon_{n}\right]$, the proof of which follows the proof of [14, Lemma 3], and taking $\tau_{n}=2 \varepsilon_{n}$.

Joint secrecy achievable rate region: We summarize the requirements in order to guarantee a reliable communication under the joint secrecy constraint as follows:

- the non-negativity for rates;

- the conditions for a reliable communication, i.e., (7);

- the conditions for the joint secrecy, i.e., (8).

That is, we have the following system of inequalities:

$$
\begin{gathered}
\sum_{j \in \mathcal{J}}\left[R_{j}+R_{j, r}\right] \leq I\left(V_{\mathcal{J}} ; Y \mid V_{\mathcal{J}^{c}}, Q\right), \quad \text { for all } \mathcal{J} \subseteq \mathcal{K}, \\
\sum_{j \in \mathcal{J}} R_{j, r} \geq I\left(V_{\mathcal{J}} ; Z \mid Q\right), \quad \text { for all } \mathcal{J} \subseteq \mathcal{K} .
\end{gathered}
$$

To obtain the desired region of $\left\{R_{i} \mid i \in \mathcal{K}\right\}$, the variables of $\left\{R_{i, r} \mid i \in \mathcal{K}\right\}$ are to be eliminated. (The rate constraints $R_{i} \geq 0$ for $i \in \mathcal{K}$ are not included here, since they will not be involved in the Fourier-Motzkin elimination. But they will be included in the final derived region.)

Fourier-Motzkin Elimination: A matrix notation of the above system of inequalities can be written as follows:

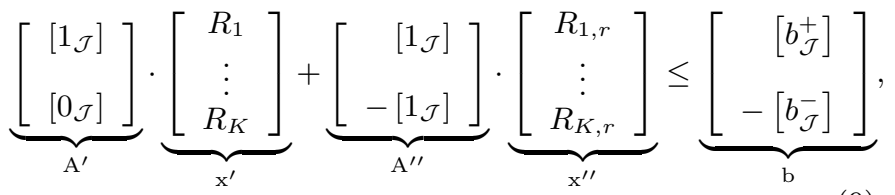

where

- $1_{\mathcal{J}}$ is the $1 \times K$ indicator vector of the subset $\mathcal{J}$ of the set $\mathcal{K}$ (which definition is given in Definition 1);

- Since $\mathcal{J}=\emptyset$ introduces only redundant inequations, only the non-empty choices of $\mathcal{J}$ need to be considered. Let $\left\{\mathcal{J}_{1}, \cdots, \mathcal{J}_{2^{K}-1}\right\}$ be the list of $2^{K}-1$ different non-empty subsets of $\mathcal{K}$. We have $\mathcal{J} \in$ $\left\{\mathcal{J}_{1}, \cdots, \mathcal{J}_{2^{K}-1}\right\}$

- $\left[1_{\mathcal{J}}\right]$ is a $\left(2^{K}-1\right) \times K$ matrix, with the $i$-th row to be the indicator vector $1_{\mathcal{J}_{i}}$, where $1 \leq i \leq 2^{K}-1$. Correspondingly,
- $\left[b_{\mathcal{J}}^{+}\right]$is a $\left(2^{K}-1\right) \times 1$ matrix with the $i$-th row to be $b_{\mathcal{J}_{i}}^{+}=I\left(V_{\mathcal{J}_{i}} ; Y \mid V_{\mathcal{J}_{i}^{c}}, Q\right)$,

- $\left[b_{\mathcal{J}}^{-}\right]$is a $\left(2^{K}-1\right) \times 1$ matrix with the $i$-th row to be $b_{\mathcal{J}_{i}}^{-}=I\left(V_{\mathcal{J}_{i}} ; Z \mid Q\right)$;

- $\left[0_{\mathcal{J}}\right]$ is a $\left(2^{K}-1\right) \times K$ matrix with zero elements.

Properties of $b_{\mathcal{J}}^{+}$and $b_{\mathcal{J}}^{-}$are given in Lemma 8 in Appendix A. which play a crucial role in removing the redundant inquations so as to avoid the double exponential complexity of the Fourier-Motzkin elimination.

Note that $\mathrm{A}^{\prime}, \mathrm{A}^{\prime \prime}$ are both $\left(2^{K+1}-2\right) \times K$ matrices. In particular, we have

$$
\mathrm{A}^{\prime \prime}=\left[\begin{array}{r}
{\left[1_{\mathcal{J}}\right]} \\
-\left[1_{\mathcal{J}}\right]
\end{array}\right]=\mathrm{B} \otimes\left[1_{\mathcal{J}}\right], \text { where } \mathrm{B}=\left[\begin{array}{r}
1 \\
-1
\end{array}\right] .
$$

Here $\otimes$ is the Kronecker product. According to 15. Theorem 1], we are looking for a $G$ such that $\left\{\mathrm{wA}^{\prime} \mathrm{x}^{\prime} \leq \mathrm{wb} \mid \mathrm{w} \in\right.$ $\mathrm{G}\}$ is equivalent to the final system of the Fourier-Motzkin elimination. Here, $\mathrm{G}$ is a base of essentially different minimal vectors of $\mathrm{F}$ that is the cone of non-negative solution $\mathrm{w} \geq 0$ of $\mathrm{wA}^{\prime \prime}=0$.

In fact, for our system defined by $(9)$, we find

$$
\mathrm{G}=\mathrm{D} \otimes \mathrm{I}_{2^{K}-1}, \quad \text { where } \mathrm{D}=\left[\begin{array}{ll}
1 & 1
\end{array}\right] .
$$

Here $\mathrm{I}_{n}$ is an $n \times n$ identity matrix. In the following, we show that $G$ (as defined in (11)) is a base of essentially different minimal vectors of $\mathrm{F}$. Note that the system defined by $\left\{\mathrm{wA}^{\prime} \mathrm{x}^{\prime} \leq \mathrm{wb} \mid \mathrm{w} \in \mathrm{G}\right\}$ is:

$$
\mathrm{GA}^{\prime} \mathrm{x}^{\prime} \leq \mathrm{Gb} \quad \Leftrightarrow \quad\left[1_{\mathcal{J}}\right] \mathrm{x}^{\prime} \leq\left[b_{\mathcal{J}}^{+}-b_{\mathcal{J}}^{-}\right] .
$$

Firstly, it is clear that $\mathrm{w} \in \mathrm{G}$ is positive, i.e., $\mathrm{w} \geq 0$.

Secondly, we show that $\mathrm{wA}^{\prime \prime}=0$ for any $\mathrm{w} \in \mathrm{G}$. Note that $\mathrm{A}^{\prime \prime}=\mathrm{B} \otimes\left[1_{\mathcal{J}}\right]$ by $(10)$. Therefore, if $\mathrm{w} \cdot\left(\mathrm{B} \otimes\left[1_{\mathcal{J}}\right]\right)=0$, we have $\mathrm{w} \cdot \mathrm{A}^{\prime \prime}=0$ as well. It is easy to verify that

$\mathrm{G} \cdot\left(\mathrm{B} \otimes\left[1_{\mathcal{J}}\right]\right)=\left(\mathrm{D} \otimes \mathrm{I}_{2^{K}-1}\right) \cdot\left(\mathrm{B} \otimes\left[1_{\mathcal{J}}\right]\right) \stackrel{(a)}{=}(\mathrm{DB}) \otimes\left[1_{\mathcal{J}}\right]=\left[0_{\mathcal{J}}\right]$,

where $(a)$ is due to the mixed-product property of the Kronecker product: $(\mathrm{A} \otimes \mathrm{B})(\mathrm{C} \otimes \mathrm{D})=(\mathrm{AC}) \otimes(\mathrm{BD})$.

Furthermore, by definition of $\mathrm{G}$ in (11), each row of $\mathrm{G}$ is essentially different from the others. And, they are all minimal according to [15, Theorem 2].

Most importantly, we show in the following that any other positive vectors $\mathrm{c}$ satisfying $\mathrm{c} \cdot \mathrm{A}^{\prime \prime}=0$, will produce only redundant inequations. For convenience, we denote $\mathrm{c}=\left[\mathrm{c}^{+} \mathrm{c}^{-}\right]$, with $\mathrm{c}^{+}=\left[c_{1}^{+}, \cdots, c_{2^{K}-1}^{+}\right]$and $\mathrm{c}^{-}=\left[c_{1}^{-}, \cdots, c_{2^{K}-1}^{-}\right]$. Then, $\mathrm{c} \cdot \mathrm{A}^{\prime \prime}=0$ is equivalent to $\mathrm{c}^{+} \cdot\left[1_{\mathcal{J}}\right]=\mathrm{c}^{-} \cdot\left[1_{\mathcal{J}}\right]$.

Recall that for $1 \leq i \leq 2^{K}-1,1_{\mathcal{J}_{i}}$ is the indicator vector of $\mathcal{J}_{i}$, and thus $c_{i}^{+}>0$ (or $c_{i}^{-}>0$ ) indicates $c_{i}^{+}$(or $c_{i}^{-}$) number of presences of $\mathcal{J}_{i}$. Let

$$
\begin{aligned}
& J_{\mathrm{c}^{+}}=\{\underbrace{\mathcal{J}_{1}, \cdots, \mathcal{J}_{1}}_{c_{1}^{+}}, \cdots, \underbrace{\mathcal{J}_{i}, \cdots, \mathcal{J}_{i}}_{c_{i}^{+}}, \cdots, \underbrace{\mathcal{J}_{2^{K}-1}, \cdots, \mathcal{J}_{2^{K}-1}}_{c_{2^{K}-1}^{+}}\} ; \\
& J_{\mathrm{c}^{-}}=\{\underbrace{\mathcal{J}_{1}, \cdots, \mathcal{J}_{1}}_{c_{1}^{-}}, \cdots, \underbrace{\mathcal{J}_{i}, \cdots, \mathcal{J}_{i}}_{c_{2^{K}-1}^{-}}, \cdots, \underbrace{\mathcal{J}_{2^{K}-1}, \cdots, \mathcal{J}_{2^{K}-1}}\} .
\end{aligned}
$$


Denote $n^{+}=\sum_{i=1}^{2^{K}-1} c_{i}^{+}$and $n^{-}=\sum_{i=1}^{2^{K}-1} c_{i}^{-}$. Simply representing $J_{\mathrm{c}^{+}}=\left\{\mathcal{J}_{1}^{+}, \mathcal{J}_{2}^{+}, \cdots, \mathcal{J}_{n^{+}}^{+}\right\}$and $J_{\mathrm{c}^{-}}=$ $\left\{\mathcal{J}_{1}^{-}, \mathcal{J}_{2}^{-}, \cdots, \mathcal{J}_{n^{-}}^{-}\right\}$, we have

$$
\begin{aligned}
& \mathrm{c}^{+} \cdot\left[1_{\mathcal{J}}\right]=\sum_{i=1}^{n^{+}} 1_{\mathcal{J}_{i}^{+}}, \quad \mathrm{c}^{-} \cdot\left[1_{\mathcal{J}}\right]=\sum_{i=1}^{n^{-}} 1_{\mathcal{J}_{i}^{-}} \\
& \mathrm{c}^{+} \cdot\left[b_{\mathcal{J}}^{+}\right]=\sum_{i=1}^{n^{+}} b_{\mathcal{J}_{i}^{+}}^{+}, \quad \mathrm{c}^{-} \cdot\left[b_{\mathcal{J}}^{-}\right]=\sum_{i=1}^{n^{-}} b_{\mathcal{J}_{i}^{-}}^{-}
\end{aligned}
$$

Note that $\mathrm{c}^{+} \cdot\left[1_{\mathcal{J}}\right]=\mathrm{c}^{-} \cdot\left[1_{\mathcal{J}}\right]$ and 13 together imply that both $J_{\mathrm{c}^{+}}$and $J_{\mathrm{c}^{-}}$share the same presence vector $c_{\#}=c^{+} \cdot\left[1_{\mathcal{J}}\right]$ (according to Definition 2). Denote the largest element of $c_{\#}$ to be $c_{\max }$.

Let $\left\{\mathcal{J}_{i}^{\oplus} \mid 1 \leq i \leq n^{+}\right\}$and $\left\{\mathcal{J}_{i}^{\ominus} \mid 1 \leq i \leq n^{-}\right\}$be the compact forms of the element rearrangement (definition of which is given in Definition 3 for $J_{\mathrm{c}^{+}}$and $J_{\mathrm{c}^{-}}$, respectively. Then, according to Lemma 5 they also share the same compact form of the element rearrangement without empty sets, i.e., $\left\{\mathcal{J}_{i}^{\oplus} \mid 1 \leq i \leq c_{\max }\right\}$. That is,

$$
\begin{aligned}
\mathcal{J}_{i}^{\oplus}=\mathcal{J}_{i}^{\ominus}, & \text { for } \quad 1 \leq i \leq c_{\max } ; \\
\mathcal{J}_{i}^{\oplus}=\mathcal{J}_{j}^{\ominus}=\emptyset, & \text { for } \quad c_{\max }<i \leq n^{+} \& c_{\max }<j \leq n^{-} .
\end{aligned}
$$

Since the element arrangement does not change the presence vector, we have by Lemma 4

$$
\mathrm{c}_{\#}=\mathrm{c}^{+} \cdot\left[1_{\mathcal{J}}\right]=\sum_{i=1}^{c_{\max }} 1_{\mathcal{J}_{i}^{\oplus}} .
$$

Now if we sum up the rows of 12 with respect to $\left\{\mathcal{J}_{i}^{\oplus} \mid 1 \leq i \leq c_{\max }\right\}$, we obtain the following inequation:

$$
\sum_{i=1}^{c_{\max }} 1_{\mathcal{J}_{i}^{\oplus}} \mathrm{x}^{\prime} \leq \sum_{i=1}^{c_{\max }}\left[b_{\mathcal{J}_{i}^{\oplus}}^{+}-b_{\mathcal{J}_{i}^{\oplus}}^{-}\right] .
$$

On the other hand, if we apply c to the original system (9), we obtain the following inequation:

$$
\begin{aligned}
\mathrm{cA}^{\prime} \mathrm{x}^{\prime} \leq \mathrm{cb} & \Rightarrow \quad \mathrm{c}^{+}\left[1_{\mathcal{J}}\right] \mathrm{x}^{\prime} \leq \mathrm{c}^{+}\left[b_{\mathcal{J}}^{+}\right]-\mathrm{c}^{-}\left[b_{\mathcal{J}}^{-}\right] \\
& \stackrel{(a)}{\Rightarrow} \quad \mathrm{c}_{\#} \mathrm{x}^{\prime} \leq \sum_{i=1}^{n^{+}} b_{\mathcal{J}_{i}^{+}}^{+}-\sum_{i=1}^{n^{-}} b_{\mathcal{J}_{i}^{-}}^{-}
\end{aligned}
$$

where $(a)$ is due to 16 and 14 .

Note that the LHS of 18 is the same as the LHS of (17) (according to (16)). However, we can show that

$$
\begin{aligned}
\text { RHS of } 18] & =\sum_{i=1}^{n^{+}} b_{\mathcal{J}_{i}^{+}}^{+}-\sum_{i=1}^{n^{-}} b_{\mathcal{J}_{i}^{-}}^{-} \stackrel{(b)}{\geq} \sum_{i=1}^{n^{+}} b_{\mathcal{J}_{i}^{\oplus}}^{+}-\sum_{i=1}^{n^{-}} b_{\mathcal{J}_{i}^{\ominus}}^{-} \\
& \left.\stackrel{(c)}{=} \sum_{i=1}^{c_{\max }}\left[b_{\mathcal{J}_{i}^{\oplus}}^{+}-b_{\mathcal{J}_{i}^{\oplus}}^{-}\right]=\text {RHS of } 17\right),
\end{aligned}
$$

where $(b)$ is according to Lemma 8 (as given in Appendix A and $(c)$ is due to (15) and $b_{\emptyset}^{+}=b_{\emptyset}^{-}=0$. That is, (18) is redundant since it is already implied by $(17)$, which can be derived as a linear combinatory with positive coefficients of inequations of 12 . Since this applies to any positive vector $c$ that is other than the rows of $G$ (as defined in (11)) such that $\mathrm{c} \cdot \mathrm{A}^{\prime \prime}=0$, therefore, the final system of (9) is equivalent to the one 12 .

As a conclusion, 12 establishes the resulting joint secrecy region.

\section{ApPENDix A \\ Properties OF $b_{\mathcal{T}}^{+}$AND $b_{\mathcal{T}}^{-}$}

Recall that $b_{\mathcal{T}}^{+}=I\left(V_{\mathcal{T}} ; Y \mid V_{\mathcal{T}^{c}}, Q\right)$ and $b_{\mathcal{T}}^{-}=I\left(V_{\mathcal{T}} ; Z \mid Q\right)$ as defined in (9). We have the following lemma:

Lemma 8. Given $\left\{\mathcal{T}_{i} \mid 1 \leq i \leq t\right\}$ as a list of $t$ subsets of $\mathcal{K}$, and its compact form of the element rearrangement $\left\{\mathcal{T}_{t, i}^{*} \mid 1 \leq i \leq t\right\}$, we have

$$
\sum_{i=1}^{t} b_{\mathcal{T}_{i}}^{+} \geq \sum_{i=1}^{t} b_{\mathcal{T}_{t, i}^{*}}^{+} \quad \text { and } \quad \sum_{i=1}^{t} b_{\mathcal{T}_{i}}^{-} \leq \sum_{i=1}^{t} b_{\mathcal{T}_{t, i}^{*}}^{-}
$$

To prove Lemma 8, we need the following two lemmas.

Lemma 9. Given $\left\{\mathcal{T}_{i} \mid 1 \leq i \leq t-1\right\}$ as a list of $t-1$ subsets of $\mathcal{K}$, and list $\left\{\mathcal{T}_{i} \mid 1 \leq i \leq t\right\}$ (with one more subset $\mathcal{T}_{t}$ included), let $\left\{\mathcal{T}_{t-1, i}^{*} \mid 1 \leq i \leq t-1\right\}$ and $\left\{\mathcal{T}_{t, i}^{*} \mid 1 \leq i \leq\right.$ $t\}$ be their compact forms of the element rearrangement, respectively. We have

$$
\mathcal{T}_{t, i}^{*}= \begin{cases}\mathcal{T}_{t-1,1}^{*} \cup \mathcal{T}_{t} & i=1 \\ \mathcal{T}_{t-1, i}^{*} \cup\left(\mathcal{T}_{t-1, i-1}^{*} \cap \mathcal{T}_{t}\right) & 1<i<t \\ \mathcal{T}_{t-1, t-1}^{*} \cap \mathcal{T}_{t} & i=t\end{cases}
$$

Proof: The proof for $i=1, t$ is straightforward by definitions of $\mathcal{T}_{t, 1}^{*}$ and $\mathcal{T}_{t, t}^{*}$. For $1<i<t$, the proof is given in steps 19)-21 (at the top of the next page), where 19] is by the definition of $\mathcal{T}_{t, i}^{*} ; 20$ is due to the distributive laws of the sets and (21) is by the definition of $\mathcal{T}_{t-1, i}^{*}$.

Lemma 10. For $\forall \mathcal{T}_{1}, \mathcal{T}_{2} \subseteq \mathcal{K}$, we have

1) $b_{\mathcal{T}_{1}}^{+}+b_{\mathcal{T}_{2}}^{+} \geq b_{\mathcal{T}_{1} \cap \mathcal{T}_{2}}^{+}+b_{\mathcal{T}_{1} \cup \mathcal{T}_{2}}^{+} ;$

2) $b_{\mathcal{T}_{1}}^{-}+b_{\mathcal{T}_{2}}^{-} \leq b_{\mathcal{T}_{1} \cap \mathcal{T}_{2}}^{-}+b_{\mathcal{T}_{1} \cup \mathcal{T}_{2}}^{-}$.

Proof: We only give the proof for $b_{\mathcal{T}_{1}}^{+}+b_{\mathcal{T}_{2}}^{+}$. A similar proof applies to $b_{\mathcal{T}_{1}}^{-}+b_{\mathcal{T}_{2}}^{-}$.

$$
\begin{aligned}
& b_{\mathcal{T}_{1}}^{+}+b_{\mathcal{T}_{2}}^{+} \stackrel{(a)}{=} I\left(V_{\mathcal{T}_{1}} ; Y \mid V_{\mathcal{T}_{1}}{ }, Q\right)+I\left(V_{\mathcal{T}_{2}} ; Y \mid V_{\mathcal{T}_{2}}, Q\right) \\
& \stackrel{(b)}{=} I\left(V_{\mathcal{T}_{1} \cap \mathcal{T}_{2}}, V_{\mathcal{T}_{1} \cap \mathcal{T}_{2}^{c}} ; Y \mid V_{\mathcal{T}_{1}}, Q\right)+I\left(V_{\mathcal{T}_{2}} ; Y \mid V_{\mathcal{T}_{2}}, Q\right) \\
& \stackrel{(c)}{=} I\left(V_{\mathcal{T}_{1} \cap \mathcal{T}_{2}^{c}} ; Y \mid V_{\mathcal{T}_{1}}^{c}, Q\right)+I\left(V_{\mathcal{T}_{1} \cap \mathcal{T}_{2}} ; Y \mid V_{\left(\mathcal{T}_{1} \cap \mathcal{T}_{2}\right)^{c}}, Q\right) \\
& +I\left(V_{\mathcal{T}_{2}} ; Y \mid V_{\mathcal{T}_{2}}, Q\right) \\
& \stackrel{(g)}{\geq} I\left(V_{\mathcal{T}_{1} \cap \mathcal{T}_{2}} ; Y \mid V_{\left(\mathcal{T}_{1} \cap \mathcal{T}_{2}\right)^{c}}, Q\right)+I\left(V_{\mathcal{T}_{1} \cap \mathcal{T}_{2}^{c}} ; Y \mid V_{\mathcal{T}_{1}{ }^{c} \cap \mathcal{T}_{2}}{ }^{c}, Q\right) \\
& +I\left(V_{\mathcal{T}_{2}} ; Y \mid V_{\mathcal{T}_{1} \cap \mathcal{T}_{2}}{ }, V_{\mathcal{T}_{1}}^{c} \cap \mathcal{T}_{2}{ }^{c}, Q\right) \\
& \stackrel{(e)}{=} I\left(V_{\mathcal{T}_{1} \cap \mathcal{T}_{2}} ; Y \mid V_{\left(\mathcal{T}_{1} \cap \mathcal{T}_{2}\right)^{c}}, Q\right)+I\left(V_{\mathcal{T}_{1} \cup \mathcal{T}_{2}} ; Y \mid V_{\left(\mathcal{T}_{1} \cup \mathcal{T}_{2}\right)^{c}}, Q\right) \text {, }
\end{aligned}
$$

where $(a)$ is by the definition of $b_{\mathcal{T}}^{+} ;(b)$ is by the fact that $\mathcal{T}_{1}=\left(\mathcal{T}_{1} \cap \mathcal{T}_{2}\right) \cup\left(\mathcal{T}_{1} \cap \mathcal{T}_{2}^{c}\right) ;(c)$ is by the chain rule of the mutual information; $(d)$ is by the facts that $\mathcal{T}_{2}^{c}=\left(\mathcal{T}_{1} \cap \mathcal{T}_{2}^{c}\right) \cup\left(\mathcal{T}_{1}^{c} \cap \mathcal{T}_{2}^{c}\right)$ and $I\left(V_{\mathcal{T}_{1} \cap \mathcal{T}_{2}^{c}} ; Y \mid V_{\mathcal{T}_{1}}{ }^{c}, Q\right)=$ $I\left(V_{\mathcal{T}_{1} \cap \mathcal{T}_{2}^{c}} ; V_{\mathcal{T}_{1}{ }^{c} \cap \mathcal{T}_{2}}, Y \mid V_{\mathcal{T}_{1}{ }^{c} \cap \mathcal{T}_{2}}{ }^{c}, Q\right) \geq I\left(V_{\mathcal{T}_{1} \cap \mathcal{T}_{2}^{c}} ; Y \mid V_{\mathcal{T}_{1}{ }^{c} \cap \mathcal{T}_{2}}{ }^{c}\right.$, 


$$
\begin{aligned}
& \mathcal{T}_{t, i}^{*}=\bigcup_{\left\{j_{1}, \ldots, j_{i}\right\} \subseteq[1: t]}\left(\bigcap_{k=1}^{i} \mathcal{T}_{j_{k}}\right)=\left\{\bigcup_{\left\{j_{1}, \ldots, j_{i}\right\} \subseteq[1: t-1]}\left(\bigcap_{k=1}^{i} \mathcal{T}_{j_{k}}\right)\right\} \bigcup\left\{\bigcup_{\left\{j_{1}, \ldots, j_{i-1}\right\} \subseteq[1: t-1]}\left(\left(\bigcap_{k=1}^{i-1} \mathcal{T}_{j_{k}}\right) \bigcap \mathcal{T}_{t}\right)\right\} \\
& =\left\{\bigcup_{\left\{j_{1}, \ldots, j_{i}\right\} \subseteq[1: t-1]}\left(\bigcap_{k=1}^{i} \mathcal{T}_{j_{k}}\right)\right\} \bigcup\left\{\left(\bigcup_{\left\{j_{1}, \ldots, j_{i-1}\right\} \subseteq[1: t-1]}\left(\bigcap_{k=1}^{i-1} \mathcal{T}_{j_{k}}\right)\right) \bigcap \mathcal{T}_{t}\right\} \\
& =\mathcal{T}_{t-1, i}^{*} \bigcup\left(\mathcal{T}_{t-1, i-1}^{*} \bigcap \mathcal{T}_{t}\right) \text {, }
\end{aligned}
$$

$Q$ ), which holds since $V_{\mathcal{T}_{1} \cap \mathcal{T}_{2}^{c}}, V_{\mathcal{T}_{1}{ }^{c} \cap \mathcal{T}_{2}}$ and $V_{\mathcal{T}_{1}{ }^{c} \cap \mathcal{T}_{2}}{ }^{c}$ are independent given $Q$; and $(e)$ is due to the facts that $\mathcal{T}_{1} \cup \mathcal{T}_{2}=\mathcal{T}_{2} \cup\left(\mathcal{T}_{1} \cap \mathcal{T}_{2}^{c}\right)$ and $\mathcal{T}_{1}{ }^{c} \cap \mathcal{T}_{2}{ }^{c}=\left(\mathcal{T}_{1} \cup \mathcal{T}_{2}\right)^{c}$.

Now we give the proof of Lemma 8 as follows.

First, we show that the statement $\sum_{i=1}^{t} b_{\mathcal{T}_{i}}^{+} \geq \sum_{i=1}^{t} b_{\mathcal{T}_{t, i}^{*}}^{+}$is true by induction.

- For $t=1$, we have by definition $\mathcal{T}_{1,1}^{*}=\mathcal{T}_{1}$. Thus, $b_{\mathcal{T}_{1}}^{+}=b_{\mathcal{T}_{1}^{*} \text { }}^{+}$and the statement is true for $t=1$.

- For $t=2$, the statement is true by Lemma 10

- Assume that the statement is true for some natural number $t-1$. That is, for any $\left\{\mathcal{T}_{i} \mid 1 \leq i \leq t-1\right\}$ as a list of $t-1$ subsets of $\mathcal{K}$, and its compact form of element rearrangement $\left\{\mathcal{T}_{t-1, i}^{*} \mid 1 \leq i \leq t-1\right\}$, we have $\sum_{i=1}^{t-1} b_{\mathcal{T}_{i}}^{+} \geq \sum_{i=1}^{t-1} b_{\mathcal{T}_{t-1, i}^{*}}^{+}$

- Now we show that the statement is also true for $t$.

$$
\begin{aligned}
& \sum_{i=1}^{t} b_{\mathcal{T}_{i}}^{+}=\sum_{i=1}^{t-1} b_{\mathcal{T}_{i}}^{+}+b_{\mathcal{T}_{t}}^{+} \stackrel{(a)}{\geq} \sum_{i=1}^{t-1} b_{\mathcal{T}_{t-1, i}^{*}}^{+}+b_{\mathcal{T}_{t}}^{+} \\
& =\sum_{i=2}^{t-1} b_{\mathcal{T}_{t-1, i}^{*}}^{+}+\left(b_{\mathcal{T}_{t-1,1}^{*}}^{+}+b_{\mathcal{T}_{t}}^{+}\right) \\
& \stackrel{\left(b_{1}\right)}{\geq} \sum_{i=2}^{t-1} b_{\mathcal{T}_{t-1, i}^{*}}^{+}+\left(b_{\mathcal{T}_{t-1,1}^{*} \cap \mathcal{T}_{t}}^{+}+b_{\mathcal{T}_{t-1,1}^{*} \cup \mathcal{T}_{t}}^{+}\right) \\
& \stackrel{\left(c_{1}\right)}{=} \sum_{i=3}^{t-1} b_{\mathcal{T}_{t-1, i}^{*}}^{+}+\left(b_{\mathcal{T}_{t-1,2}^{*}}^{+}+b_{\mathcal{T}_{t-1,1}^{*} \cap \mathcal{T}_{t}}^{+}\right)+b_{\mathcal{T}_{t, 1}^{*}}^{+} \\
& \stackrel{\left(b_{2}\right)}{\geq} \sum_{i=3}^{t-1} b_{\mathcal{T}_{t-1, i}^{*}}^{+}+\left(b_{\mathcal{T}_{t-1,2}^{*} \cap \mathcal{T}_{t}}^{+}+b_{\mathcal{T}_{t-1,2}^{*}}^{+} \cup\left(\mathcal{T}_{t-1,1}^{*} \cap \mathcal{T}_{t}\right)\right) \\
& +b_{\mathcal{T}_{t, 1}^{*}}^{+} \\
& \stackrel{\left(c_{2}\right)}{=} \sum_{i=4}^{t-1} b_{\mathcal{T}_{t-1, i}^{*}}^{+}+\left(b_{\mathcal{T}_{t-1,3}^{*}}^{+}+b_{\mathcal{T}_{t-1,2}^{*} \cap \mathcal{T}_{t}}^{+}\right)+\sum_{i=1}^{2} b_{\mathcal{T}_{t, i}^{*}}^{+} \\
& \text {: }
\end{aligned}
$$

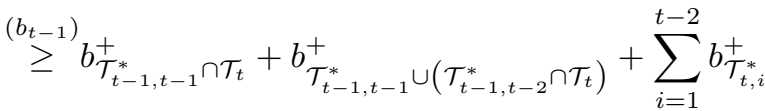

$$
\begin{aligned}
& \stackrel{\left(c_{t-1}\right)}{=} \sum_{i=1}^{t} b_{\mathcal{T}_{t, i}^{*}}^{+}
\end{aligned}
$$

where $(a)$ is due to the fact that the statement is true for $t-1$; and for $1 \leq j \leq t-1$, step $\left(b_{j}\right)$ is by applying
Lemma 10 and the fact that $\mathcal{T}_{t-1, j}^{*} \cap\left(\mathcal{T}_{t-1, j-1}^{*} \cap \mathcal{T}_{t}\right)=$ $\mathcal{T}_{t-1, j}^{*} \cap \mathcal{T}_{t}$ for $2 \leq j \leq t-1$ (since $\mathcal{T}_{t-1, j}^{*} \subseteq \mathcal{T}_{t-1, j-1}^{*}$ by definition); step $\left(c_{j}\right)$ is by applying Lemma 9 . In particular, $\left(c_{1}\right)$ is by the fact that $\mathcal{T}_{t, 1}^{*}=\mathcal{T}_{t-1,1}^{*} \bigcup \mathcal{T}_{t}$; step $\left(c_{t-1}\right)$ is by the fact that $\mathcal{T}_{t, t}^{*}=\mathcal{T}_{t-1, t-1}^{*} \cap \mathcal{T}_{t}$; and other intermediate steps are by the fact that $\mathcal{T}_{t-1, j}^{*} \cup$ $\left(\mathcal{T}_{t-1, j-1}^{*} \cap \mathcal{T}_{t}\right)=\mathcal{T}_{t, j}^{*}$ for $1<j<t$.

A similar proof can be applied to show that the statement for $\sum_{i=1}^{t} b_{\mathcal{T}_{i}}^{-}$is also true. This concludes our proof.

\section{REFERENCES}

[1] R. Ahlswede, Multi-way communication channels. Akadémiai Kiadó, 1973.

[2] H. H.-J. Liao, Multiple Access Channels. Honolulu: Ph.D. Dissertation, University of Hawaii, 1972.

[3] A. D. Wyner, "The wire-tap channel," Bell Syst. Tech. J., vol. 54 , no. 8, pp. $1355-1387$, Oct. 1975.

[4] I. Csiszár and J. Körner, "Broadcast channels with confidential messages," IEEE Trans. Inf. Theory, vol. 24, no. 3, pp. 339-348, May 1978.

[5] E. Tekin and A. Yener, "The gaussian multiple access wire-tap channel," IEEE Trans. Inf. Theory, vol. 54, no. 12, pp. 57475755, Dec. 2008.

[6] X. Tang, R. Liu, P. Spasojevic, and H. Poor, "Multiple access channels with generalized feedback and confidential messages," in Proc. 2007 IEEE Information Theory Workshop (ITW 2007), Sept. 2007, pp. 608-613.

[7] E. Tekin and A. Yener, "The general gaussian multiple-access and two-way wiretap channels: Achievable rates and cooperative jamming," IEEE Trans. Inf. Theory, vol. 54, no. 6, pp. 27352751, Jun. 2008.

[8] E. Ekrem and S. Ulukus, "On the secrecy of multiple access wiretap channel," in Proc. 2008 46th Annual Allerton Conference on Communication, Control, and Computing, Sept. 2008, pp. 1014-1021.

[9] O. O. Koyluoglu and H. El Gamal, "Cooperative encoding for secrecy in interference channels," IEEE Trans. Inf. Theory, vol. 57, no. 9, pp. 5682-5694, Sept. 2011.

[10] M. Wiese and H. Boche, "Strong secrecy for multiple access channels," in Information Theory, Combinatorics, and Search Theory, ser. Lecture Notes in Computer Science. Springer Berlin Heidelberg, 2013, vol. 7777, pp. 71-122.

[11] Y.-K. Chia and A. El Gamal, "Three-receiver broadcast channels with common and confidential messages," IEEE Trans. Inf. Theory, vol. 58, no. 5, pp. 2748-2765, May 2012.

[12] Y. Chen, O. O. Koyluoglu, and H. Vinck, "On secure communication over the multiple access channel," in Proc. 2016 IEEE International Symposium on Information Theory and Its Applications (ISITA 2016), Oct. 2016.

[13] A. E. Gamal and Y.-H. Kim, Network Information Theory. New York, NY, USA: Cambridge University Press, 2012.

[14] R. Liu, I. Maric, P. Spasojević, and R. Yates, "Discrete memoryless interference and broadcast channels with confidential messages: Secrecy rate regions," IEEE Trans. Inf. Theory, vol. 54, no. 6, pp. 2493-2507, Jun. 2008.

[15] J. louis Imbert, "Fourier's elimination: Which to choose?" 1993. 